First published in:

\title{
Non-smooth and time-varying systems of geometrically nonlinear beams
}

\author{
Pierre Barthels*, Jörg Wauer \\ Institut für Technische Mechanik, Universität Karlsruhe, 76128 Karlsruhe, Germany
}

Accepted 7 January 2008

The peer review of this article was organised by the Guest Editor

Available online 4 March 2008

\begin{abstract}
Bending vibrations of geometrically nonlinear beams, which are connected with some clearance in their contact areas, are analyzed during dynamic extending and retracting motion of the different segments. For the physical model of a fork lifter, as an example of application, the governing system equations are derived by applying Hamilton's principle. Using a discretization procedure, based on admissible shape functions, a system of coupled, nonlinear, time-varying, ordinary differential equations is generated. Linearization and model reduction leads to a sequence of simple models. On the basis of these models, an adaptive state regulator and an adaptive full-state observer (Luenberger Observer) are designed for vibration suppression using the optimal linear quadratic regulator $(L Q R)$. The adaptive controller and observer are applied to the original, significantly more complicated, geometrically nonlinear and time-varying system with clearance so that the robustness of the controlled system can be studied during dynamic extending and retracting motions.
\end{abstract}

(C) 2008 Elsevier Ltd. All rights reserved.

\section{Introduction}

Many technical devices contain slidable systems of structural components with clearance as, e.g., the ladder of a ladder truck or the telescopic mast of a fork lifter (see Fig. 1). Due to the required lightweight construction of the extending and retracting segments of these systems, bending vibrations occur, which have to be suppressed by active control.

A first step to develop a controller concept for prevention from harmful vibrations is an appropriate modeling of such systems. In many publications, e.g. Ref. [1], a multi-body approach is used. This contribution presents an alternative approach, using a fork lifter with a two-sectional telescopic mast as an example of application. The two segments of the mast are modeled as flexible beams. The clearance, which always exists in such systems, produces nonlinear effects and the different segments are pre-stressed due to their self-weight and the weight of the transported good. In Ref. [2] the modeling is shown for systems with a non-eccentric load. The different segments are supposed to be slender and modeled as Bernoulli/Euler beams. In the present contribution the load is supposed to act eccentrically on the system (see Fig. 1) and for large

*Corresponding author.

E-mail addresses: barthels@itm.uka.de (P. Barthels), wauer@itm.uka.de (J. Wauer). 

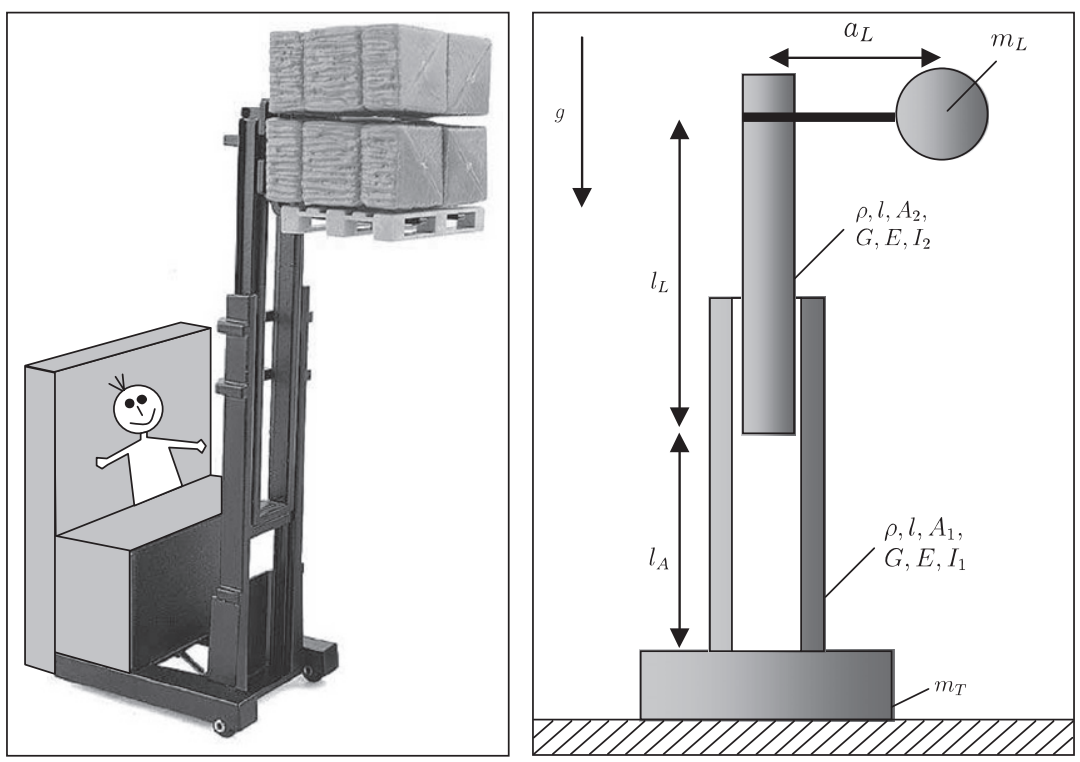

Fig. 1. Fork lifter and system model.

deformations, the beams are modeled as geometrically nonlinear Timoshenko beams. The discrete contact forces between the different segments are realized via spring-damper systems. Compared to Ref. [3], where the telescopic motion is supposed to be quasi-static, the dynamics of the extending and retracting motion of the different segments is taken into account in this contribution.

\section{Physical model}

The procedure is illustrated in Fig. 2 for the considered two-section telescopic beam system. Beam 1 is fixed at a rigid vehicle unit with the prescribed displacement $u_{0}(t)$. Beam 2 carries an eccentric point load. The deformation of the beams is represented by the horizontal displacements $u_{1}\left(z_{1}, t\right)$ and $u_{2}\left(z_{2}, t\right)$, the vertical displacements $w_{1}\left(z_{1}, t\right)$ and $w_{2}\left(z_{2}, t\right)$ and the angles $\alpha_{1}\left(z_{1}, t\right)$ and $\alpha_{2}\left(z_{2}, t\right)$. Assuming that the longitudinal axes of the beams are inextensible, one obtains the kinematic relations

$$
w_{j, z_{j}}=-1+\sqrt{1-u_{j, z_{j}}^{2}} \quad(j=1,2)
$$

between the horizontal displacements $u_{j}\left(z_{j}, t\right)$ and the vertical displacements $w_{j}\left(z_{j}, t\right)(j=1,2)$.

The model is specified by the following parameters: length $l$ of the beams (to be equal in most practical applications), constant cross-sectional areas $A_{1,2}$, constant cross-sectional moments of inertia $I_{1,2}$, density $\rho$, Young's modulus $E$ and shear modulus $G$ of the two flexible components, mass $m_{L}$ and eccentricity $a_{L}$ of the load, mass of the vehicle $m_{T}$ and given telescopic lengths $l_{A}(t)$ and $l_{L}(t)$. The contact is defined by the given number $n$ of contact points, the clearance $l_{S}$, spring stiffness $c$, and damping coefficient $d$. In the axial direction it is assumed that there is no friction and that the entire force flow goes from the upper part into the lower part through the lowest contact point.

\section{Formulation}

\subsection{Boundary value problem}

The system equations are derived by applying the extended Hamilton's principle

$$
\delta \int_{t_{0}}^{t_{1}}(T-V) \mathrm{d} t+\int_{t_{0}}^{t_{1}} W_{\text {virt }} \mathrm{d} t=0
$$




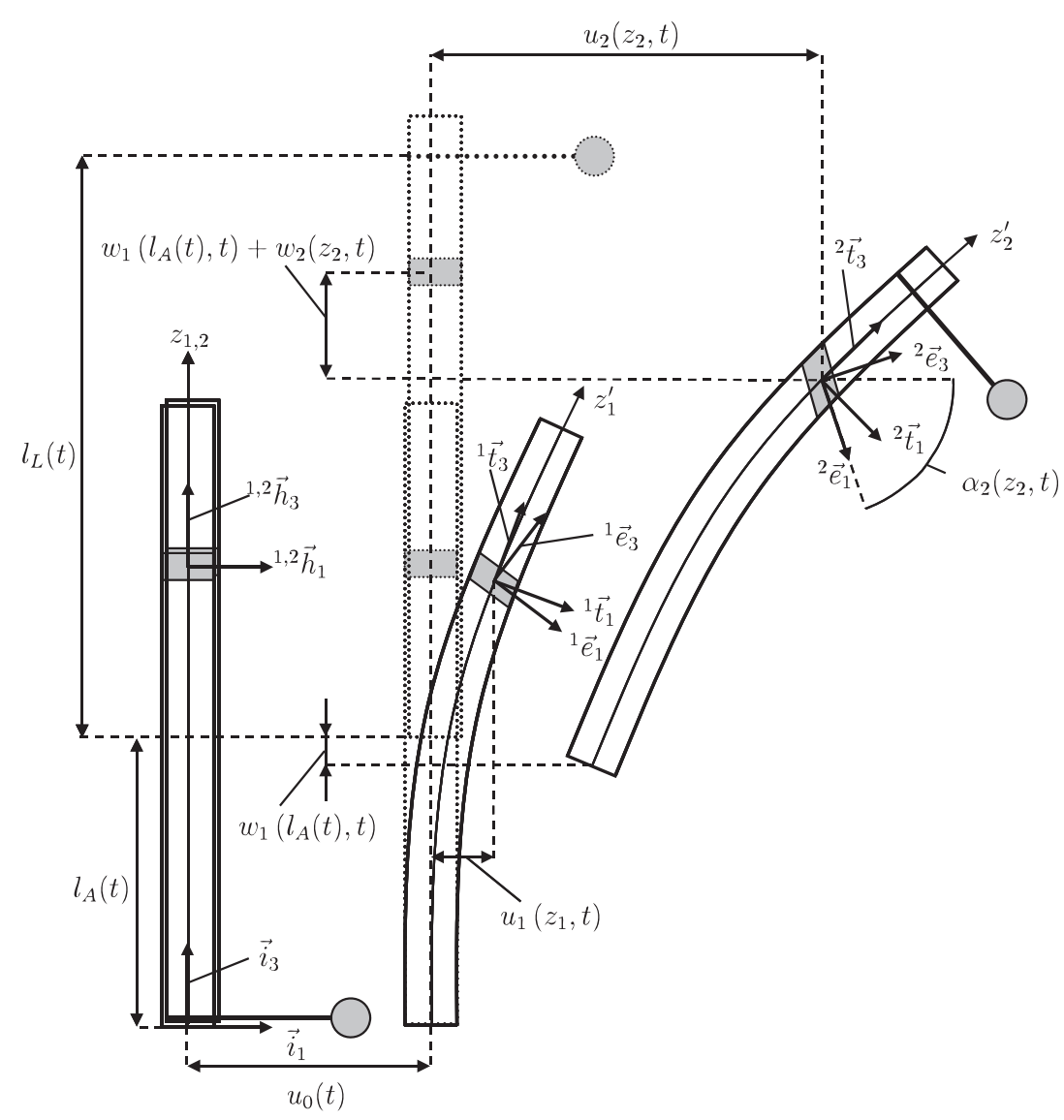

Fig. 2. Model in undeformed and deformed state.

where $T$ is the kinetic energy, $V$ is the potential energy and $W_{\text {virt }}$ is the virtual work of the non-conservative forces of the considered system. The potential energy $V$ is divided into an elastic part $V_{e}$ which is derived using Green's nonlinear strain tensor and into $V_{g}$, which is due to gravity. It is assumed that there is no warping of the cross-sectional areas which are supposed to be symmetrical and that the vertical velocities $w_{1, t}$ and $w_{2, t}$ are negligible for the kinetic energy of the system. The elastic part $V_{e}$ of the potential energy reads in a third-order approximation for each of the two beams

$$
V_{e j}^{(3)}=\int_{0}^{l}\left(\frac{G A_{j}}{2}\left(u_{j, z_{j}}-\alpha_{j}\right)^{2}+\frac{E I_{j}}{2} \alpha_{j, z_{j}}^{2}\right) \mathrm{d} z_{j} \quad(j=1,2)
$$

and the potential energy $V_{g}$, which is due to gravity, reads in a third-order approximation

$$
V_{g 1}^{(3)}=\frac{l}{2} \rho A_{1} l g-\frac{1}{2} \rho A_{1} g \int_{0}^{l}\left(l-z_{1}\right) u_{1, z_{1}}^{2} \mathrm{~d} z_{1}
$$

for the first beam,

$$
V_{g 2}^{(3)}=\left(l_{A}(t)+\frac{l}{2}\right) \rho A_{2} l g-\frac{1}{2} \rho A_{2} g\left(l \int_{0}^{l_{A}} u_{1, z_{1}}^{2} \mathrm{~d} z_{1}+\int_{0}^{l}\left(l-z_{2}\right) u_{2, z_{2}}^{2} \mathrm{~d} z_{2}\right)
$$


for the second beam and

$$
\begin{aligned}
V_{g L}^{(3)}= & m_{L} g\left(l_{A}(t)+l_{L}(t)\right)-\frac{1}{2} m_{L} g \int_{0}^{l_{A}} u_{1, z_{1}}^{2} \mathrm{~d} z_{1}-\frac{1}{2} m_{L} g \int_{0}^{l_{L}} u_{2, z_{2}}^{2} \mathrm{~d} z_{2} \\
& -m_{L} g a_{L}\left(\alpha_{2}\left(l_{L}(t), t\right)-\frac{1}{6} \alpha_{2}^{3}\left(l_{L}(t), t\right)\right)
\end{aligned}
$$

for the load. For the kinetic energy one obtains

$$
T_{1}=\frac{1}{2} \rho I_{1} \int_{0}^{l} \alpha_{1, t}^{2} \mathrm{~d} z_{1}+\frac{1}{2} \rho A_{1} \int_{0}^{l}\left(u_{0, t}+u_{1, t}\right)^{2} \mathrm{~d} z_{1}
$$

for the first beam,

$$
\begin{aligned}
T_{2}= & \frac{1}{2} \rho I_{2} \int_{0}^{l} \alpha_{2, t}^{2} \mathrm{~d} z_{2} \\
& +\frac{1}{2} \rho A_{2} \int_{0}^{l}\left(\left(u_{0, t}+u_{2, t}\right)^{2}+\left(1-u_{1, z_{1}}^{2}\left(l_{A}(t), t\right)\right) l_{A, t}^{2}\right) \mathrm{d} z_{2}
\end{aligned}
$$

for the second beam,

$$
\begin{aligned}
T_{L}^{(3)}= & \frac{1}{2} m_{L}\left(\left(u_{0, t}+u_{2, z_{2}}\left(l_{L}(t), t\right) l_{L, t}+u_{2, t}\left(l_{L}(t), t\right)\right)^{2}\right. \\
& -2 a_{L} \alpha_{2}\left(l_{L}(t), t\right)\left(u_{0, t}+u_{2, z_{2}}\left(l_{L}(t), t\right) l_{L, t}+u_{2, t}\left(l_{L}(t), t\right)\right) \\
& \times\left(\alpha_{2, z_{2}}\left(l_{L}(t), t\right) l_{L, t}+\alpha_{2, t}\left(l_{L}(t), t\right)\right) \\
& +\left[l_{A, t}+l_{L, t}-a_{L}\left(\alpha_{2, z_{2}}\left(l_{L}(t), t\right) l_{L, t}+\alpha_{2, t}\left(l_{L}(t), t\right)\right)\right]^{2} \\
& -\left(u_{1, z_{1}}^{2}\left(l_{A}(t), t\right) l_{A, t}+u_{2, z_{2}}^{2}\left(l_{L}(t), t\right) l_{L, t}\right) \\
& \times\left[l_{A, t}+l_{L, t}-a_{L}\left(\alpha_{2, z_{2}}\left(l_{L}(t), t\right) l_{L, t}+\alpha_{2, t}\left(l_{L}(t), t\right)\right)\right] \\
& \left.+a_{L} \alpha_{2}^{2}\left(l_{L}(t), t\right)\left(l_{A, t}+l_{L, t}\right)\left(\alpha_{2, z_{2}}\left(l_{L}(t), t\right) l_{L, t}+\alpha_{2, t}\left(l_{L}(t), t\right)\right)\right)
\end{aligned}
$$

for the load (in a third-order approximation) and

$$
T_{T}=\frac{1}{2} m_{T} u_{0, t}^{2}
$$

for the vehicle unit. Since no internal damping of the beam segments will be taken into consideration, as the worst case for control, the virtual work $W_{\text {virt }}$ contains the contact forces between the beams, the driving force of the vehicle $F_{T}$ and the force $F_{R}$ which is applied on the lower segment of the system at $z_{1}=l_{R}$ by an actuator:

$$
\begin{aligned}
W_{\mathrm{virt}}= & \sum_{i=1}^{n}\left(\delta u_{0}+\delta u_{1}\left(\left(l-l_{A}(t)\right)\left(\frac{i-1}{n-1}\right)+l_{A}(t), t\right)\right) F_{K, i} \\
& -\sum_{i=1}^{n}\left(\delta u_{0}+\delta u_{2}\left(\left(l-l_{A}(t)\right)\left(\frac{i-1}{n-1}\right), t\right)\right) F_{K, i} \\
& +\delta u_{0}(t) F_{T}+\delta u_{1}\left(l_{R}, t\right) F_{R} .
\end{aligned}
$$

The contact forces $F_{K, i}(i=1 . . n)$ in Eq. (11) are realized via spring-damper systems

$$
F_{K, i}=C_{K}\left(u_{i}^{*}\right)+u_{i, t}^{*} D_{K}\left(u_{i}^{*}\right)
$$




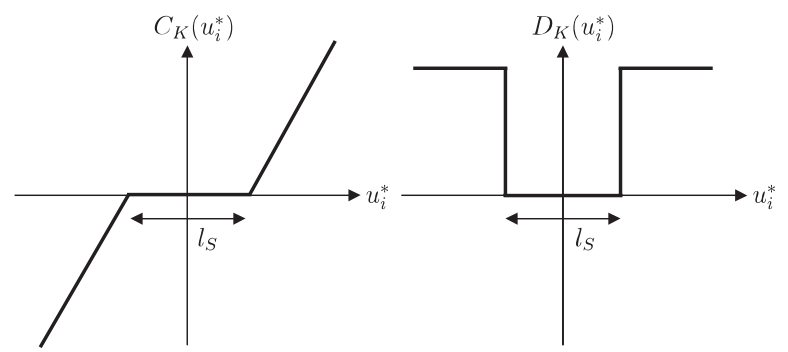

Fig. 3. Spring and damping characteristic of the contact formulation.

with (see Fig. 3)

$$
\begin{gathered}
C_{K}\left(u_{i}^{*}\right)=c\left[u_{i}^{*}-\frac{1}{2}\left(u_{i}^{*}+\frac{l_{S}}{2}\right) \operatorname{sign}\left(u_{i}^{*}+\frac{l_{S}}{2}\right)+\frac{1}{2}\left(u_{i}^{*}-\frac{l_{S}}{2}\right) \operatorname{sign}\left(u_{i}^{*}-\frac{l_{S}}{2}\right)\right], \\
D_{K}\left(u_{i}^{*}\right)=d\left[1-\frac{1}{2} \operatorname{sign}\left(u_{i}^{*}+\frac{l_{S}}{2}\right)+\frac{1}{2} \operatorname{sign}\left(u_{i}^{*}-\frac{l_{S}}{2}\right)\right]
\end{gathered}
$$

and

$$
u_{i}^{*}(t)=u_{2}\left(\left(l-l_{A}(t)\right)\left(\frac{i-1}{n-1}\right), t\right)-u_{1}\left(\left(l-l_{A}(t)\right)\left(\frac{i-1}{n-1}\right)+l_{A}(t), t\right) .
$$

In Eqs. (13)-(15), $n$ is the number of contact points, $l_{S}$ denotes the clearance, $c$ is a spring stiffness, and $d$ is a damping coefficient.

\subsection{Discretization}

A classical discretization procedure based on admissible shape functions

$$
\begin{gathered}
\bar{u}_{1}\left(z_{1}, t\right)=\sum_{i=1}^{N_{1}} q_{i}(t) U_{1_{i}}\left(z_{1}\right), \\
\bar{\alpha}_{1}\left(z_{1}, t\right)=\sum_{i=1}^{N_{1}} q_{i}(t) \Phi_{1_{i}}\left(z_{1}\right), \\
\bar{u}_{2}\left(z_{2}, t\right)=\sum_{i=1}^{N_{2}} q_{N_{1}+i}(t) U_{2_{i}}\left(z_{2}\right)
\end{gathered}
$$

and

$$
\bar{\alpha}_{2}\left(z_{2}, t\right)=\sum_{i=1}^{N_{2}} q_{N_{1}+i}(t) \Phi_{2_{i}}\left(z_{2}\right)
$$

is used to generate system equations of the type

$$
\begin{aligned}
& \delta \mathbf{q}^{\mathrm{T}}\left\{\mathbf{M}\left(\mathbf{q}, l_{L}\right) \ddot{\mathbf{q}}-\mathbf{f}\left(\mathbf{q}, \dot{\mathbf{q}}, u_{0, t}, u_{0, t t}, l_{A}, l_{A, t}, l_{A, t t}, l_{L}, l_{L, t}, l_{L, t t}, F_{R}\right)\right\} \\
& +\delta u_{0}\left\{\left(m_{1}+m_{2}+m_{L}+m_{T}\right) u_{0, t t}-f\left(\mathbf{q}, \dot{\mathbf{q}}, \ddot{\mathbf{q}}, l_{L, t}, l_{L, t t}, F_{T}\right)\right\}=0 .
\end{aligned}
$$

Appropriate shape functions are modes of a clamped-free Timoshenko beam (segment 1) and of a free-free Timoshenko beam (segment 2). 
As the motion of the vehicle unit is assumed to be a prescribed function of the time in this contribution, one obtains

$$
\delta u_{0}=0
$$

and through this a coupled system of nonlinear, time-varying, ordinary differential equations of the type

$$
\mathbf{M}\left(\mathbf{q}, l_{L}\right) \ddot{\mathbf{q}}=\mathbf{f}\left(\mathbf{q}, \dot{\mathbf{q}}, u_{0, t}, u_{0, t t}, l_{A}, l_{A, t}, l_{A, t t}, l_{L}, l_{L, t}, l_{L, t t}, F_{R}\right),
$$

as the mathematical model.

\section{Vibration suppression concept}

To suppress vibrations, a state space control concept is introduced with a collocated actuator-sensor pair at $z_{1}=l_{R}$, which is supposed to be ideal in this contribution. For fixed telescopic lengths $l_{A}=$ const. and $l_{L}=$ const. and with $F_{R}=0$ and $u_{0}=$ const., the system (Eq. (22)) is time-invariant and can be linearized about its static equilibrium position $\mathbf{q}_{0}$. The coordinate transformation $\mathbf{q}_{l}=\mathbf{q}-\mathbf{q}_{0}$ leads to

$$
\mathbf{M} \ddot{\mathbf{q}}_{l}+\mathbf{C} \mathbf{q}_{l}=\mathbf{b}_{R}^{*} F_{R}+\mathbf{b}_{0}^{*} u_{0, t t},
$$

with the mass matrix $\mathbf{M}$ the stiffness matrix $\mathbf{C}$ and the input matrices $\mathbf{b}_{R}^{*}$ and $\mathbf{b}_{0}^{*}$. The displacement of the first segment at $z_{1}=l_{R}$ reads

$$
\bar{u}_{1}\left(l_{R}, t\right)=\left[U_{1_{1}}\left(l_{R}\right), \ldots, U_{1_{N}}\left(l_{R}\right), 0, \ldots, 0\right]\left(\mathbf{q}_{l}+\mathbf{q}_{0}\right)=\mathbf{c}_{R}^{*^{\mathrm{T}}} \mathbf{q}_{l}+u_{R_{0}} .
$$

\subsection{Validation of the mathematical model}

The linearized mathematical model (Eq. (23)) is validated for a system with a fixed position of the vehicle unit, which is defined by the following parameters: $l=1.35 \mathrm{~m}, A_{1,2}=0.001 \mathrm{~m}^{2}, I_{1,2}=0.83 \times 10^{-8} \mathrm{~m}^{4}$, $\rho=7850 \mathrm{~kg} / \mathrm{m}^{3}, \quad E=2.1 \times 10^{11} \mathrm{~N} / \mathrm{m}^{2}, \quad G=0.8 \times 10^{11} \mathrm{~N} / \mathrm{m}^{2}, \quad m_{L}=17.897 \mathrm{~kg}, \quad a_{L}=0.5 \mathrm{~m}, \quad l_{S}=0 \mathrm{~m}$, $c=10^{8} \mathrm{~N} / \mathrm{m}, d=0 \mathrm{Ns} / \mathrm{m}, n=3, N_{1}=4, N_{2}=6$. The validation takes place by comparing the natural frequencies of the linearized model (Eq. (23)) to those of a corresponding FE-model (ANSYS) with 5400 SOLID45-elements, 2 MASS21-elements (load and vehicle unit) and 8976 nodes. The first three natural frequencies are plotted versus the telescopic length $l_{T}=l_{A}+l_{L}$ (with $l_{A}=0$ for $0 \leqslant l_{L}<l$ ) in Figs. 4-6.

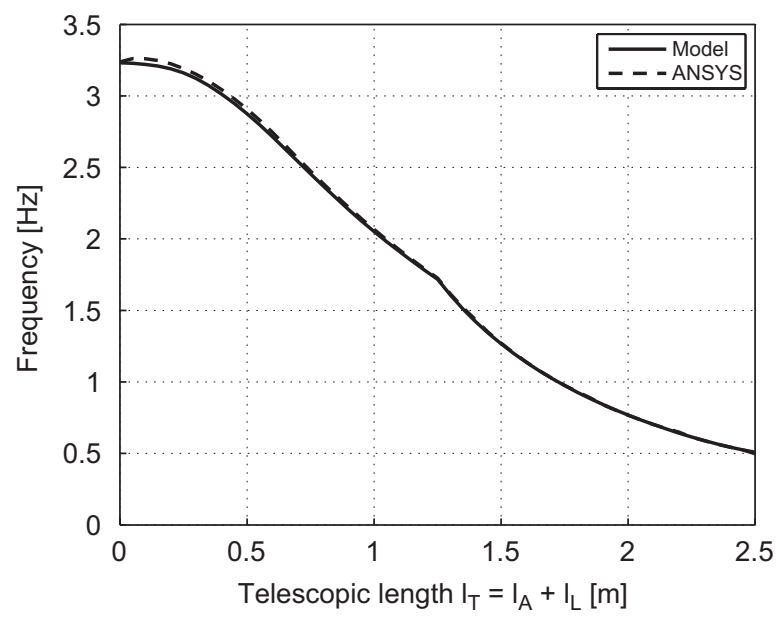

Fig. 4. Natural frequency 1. 


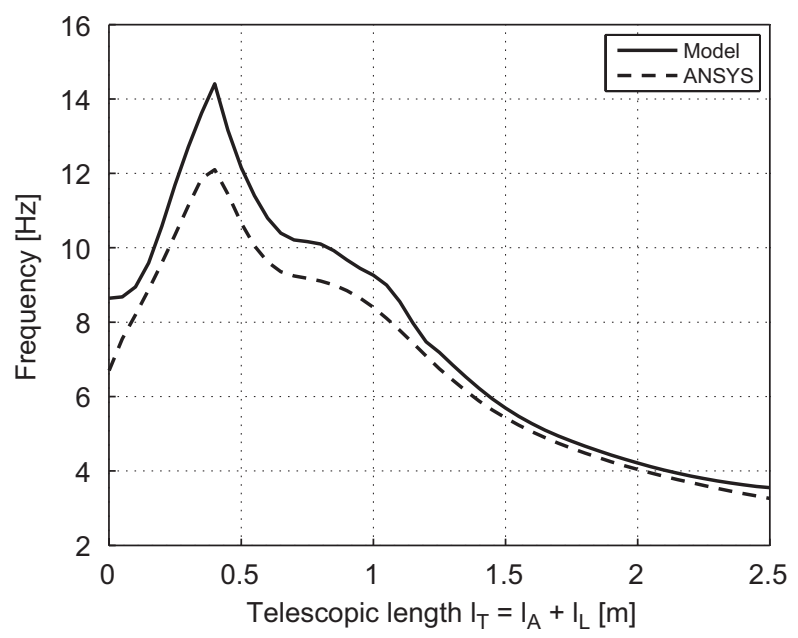

Fig. 5. Natural frequency 2.

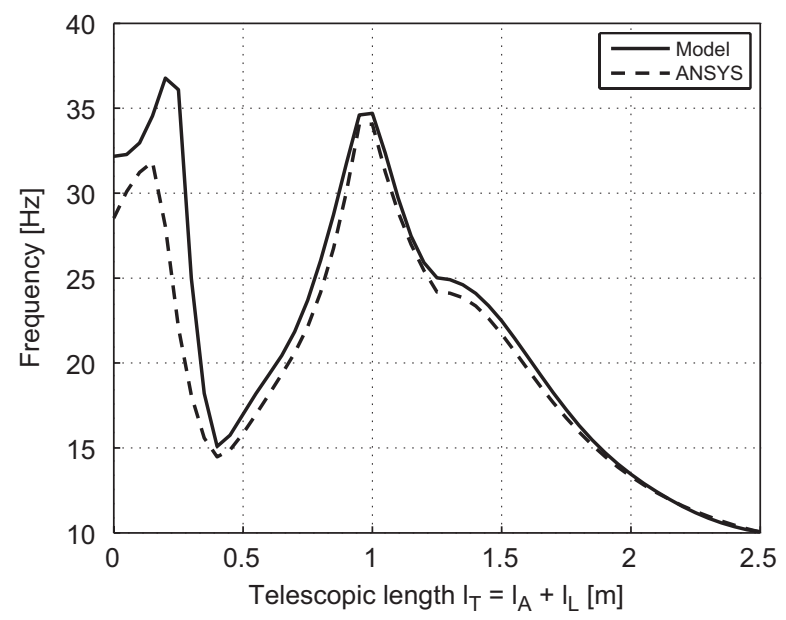

Fig. 6. Natural frequency 3.

\subsection{Model reduction}

Eq. (23) represents a $\left(N_{1}+N_{2}\right)$-degree-of-freedom system. The objective of an order reduction is to find, for a given model of high order, a model of significantly lower order whose dynamic behavior approximates the original behavior as well as possible in a specified frequency bandwidth. For this purpose, the system equations are transformed to modal coordinates according to

$$
\mathbf{q}_{l}=\Theta \mathbf{y}
$$

where $\mathbf{y}$ is the vector of modal amplitudes and $\boldsymbol{\Theta}$ is the matrix of normalized eigenvectors $\boldsymbol{\theta}_{i}\left(i=1 . . N_{1}+N_{2}\right)$ ordered by increasing natural frequencies $\left(\omega_{1} \leqslant \omega_{2} \leqslant \cdots \leqslant \omega_{N_{1}+N_{2}}\right)$. With

$$
\boldsymbol{\Theta}^{\mathrm{T}} \mathbf{M \Theta}=\mathbf{I}
$$

and

$$
\boldsymbol{\Theta}^{\mathrm{T}} \mathbf{C} \boldsymbol{\Theta}=\operatorname{diag}\left(\omega_{i}^{2}\right)=\boldsymbol{\Omega}
$$


one obtains

$$
\underbrace{\boldsymbol{\Theta}^{\mathrm{T}} \mathbf{M} \boldsymbol{\Theta}}_{\mathbf{I}} \ddot{\mathbf{y}}+\underbrace{\boldsymbol{\Theta}^{\mathrm{T}} \mathbf{C} \boldsymbol{\Theta}}_{\boldsymbol{\Omega}} \mathbf{y}=\underbrace{\boldsymbol{\Theta}^{\mathrm{T}} \mathbf{b}_{R}^{*}}_{\mathbf{b}_{R}^{* *}} F_{R}+\underbrace{\boldsymbol{\Theta}^{\mathrm{T}} \mathbf{b}_{0}^{*}}_{\mathbf{b}_{0}^{* *}} u_{0, t t}
$$

and

$$
\bar{u}_{1}\left(l_{R}, t\right)=\mathbf{c}_{R}^{*^{\mathrm{T}}} \boldsymbol{\Theta} \mathbf{y}+u_{R_{0}}=\mathbf{c}_{R}^{* *^{\mathrm{T}}} \mathbf{y}+u_{R_{0}} .
$$

If the modes are divided into low-frequency modes which respond dynamically, and high-frequency modes which respond statically, the reduced model reads

$$
\left(\begin{array}{cc}
\mathbf{I} & \mathbf{0} \\
\mathbf{0} & \mathbf{0}
\end{array}\right)\left(\begin{array}{c}
\ddot{\mathbf{y}}_{r} \\
\ddot{\mathbf{y}}_{h}
\end{array}\right)+\left(\begin{array}{cc}
\boldsymbol{\Omega}_{r} & \mathbf{0} \\
\mathbf{0} & \boldsymbol{\Omega}_{h}
\end{array}\right)\left(\begin{array}{c}
\mathbf{y}_{r} \\
\mathbf{y}_{h}
\end{array}\right)=\left(\begin{array}{c}
\mathbf{b}_{R r}^{* *} \\
\mathbf{b}_{R h}^{* *}
\end{array}\right) F_{R}+\left(\begin{array}{c}
\mathbf{b}_{0 r}^{* *} \\
\mathbf{b}_{0 h}^{* *}
\end{array}\right) u_{0, t t},
$$

neglecting the modal mass of the high-frequency modes. With

$$
\bar{u}_{1}\left(l_{R}, t\right)=\mathbf{c}_{R r}^{* *^{\mathrm{T}}} \mathbf{y}_{r}+\mathbf{c}_{R h}^{* *^{\mathrm{T}}} \mathbf{y}_{h}+u_{R_{0}}
$$

the reduced state space model finally reads

$$
\begin{gathered}
\left(\begin{array}{c}
\dot{\mathbf{y}}_{r} \\
\ddot{\mathbf{y}}_{r}
\end{array}\right)=\underbrace{\left(\begin{array}{cc}
\mathbf{0} & \mathbf{I} \\
-\boldsymbol{\Omega}_{r} & \mathbf{0}
\end{array}\right)}_{\mathbf{\mathbf { z }}} \underbrace{\left(\begin{array}{c}
\mathbf{y}_{r} \\
\dot{\mathbf{y}}_{r}
\end{array}\right)}_{\mathbf{z}}+\underbrace{\left(\begin{array}{c}
\mathbf{0} \\
\mathbf{b}_{R r}^{* * t}
\end{array}\right)}_{\mathbf{b}_{R}} F_{R}+\underbrace{\left(\begin{array}{c}
\mathbf{0} \\
\mathbf{b}_{0 r}^{* *}
\end{array}\right)}_{\mathbf{b}_{0}} u_{0, t t}, \\
\bar{u}_{1}\left(l_{R}, t\right)=\underbrace{\left[\mathbf{c}_{R r}^{* * \mathrm{~T}}, 0, \ldots, 0\right]}_{\mathbf{c}_{R}^{\mathrm{T}}} \mathbf{z}+\underbrace{\mathbf{c}_{R h}^{* * \mathrm{~T}} \boldsymbol{\Omega}_{h}^{-1} \mathbf{b}_{R h}^{* *}}_{d_{R}} F_{R}+\underbrace{\mathbf{c}_{R h}^{* * \mathrm{~T}} \boldsymbol{\Omega}_{h}^{-1} \mathbf{b}_{0 h}^{* *}}_{d_{0}} u_{0, t t}+u_{R_{0}} .
\end{gathered}
$$

\subsection{Linear quadratic regulator}

The idea within the state space approach using the optimal linear quadratic regulator $(L Q R)$ is to seek a linear state feedback with constant gain $\mathbf{r}$, i.e.,

$$
F_{R}=-\mathbf{r}^{\mathrm{T}} \mathbf{z}
$$

such that the following quadratic cost function is minimized:

$$
J=\int_{0}^{\infty}\left(\mathbf{z}^{\mathrm{T}} \mathbf{Q} \mathbf{z}+\frac{1}{\kappa} F_{R}^{2}+2 \mathbf{z}^{\mathrm{T}} \mathbf{n} F_{R}\right) \mathrm{d} t .
$$

The solution of this problem is

$$
\mathbf{r}^{\mathrm{T}}=\kappa\left(\mathbf{b}_{R}^{\mathrm{T}} \mathbf{P}+\mathbf{n}^{\mathrm{T}}\right)
$$

where $\mathbf{P}$ is the symmetric positive definite solution of the algebraic Riccati equation

$$
\mathbf{P A}+\mathbf{A}^{\mathrm{T}} \mathbf{P}+\mathbf{Q}-\kappa\left(\mathbf{P} \mathbf{b}_{R}+\mathbf{n}\right)\left(\mathbf{b}_{R}^{\mathrm{T}} \mathbf{P}+\mathbf{n}^{\mathrm{T}}\right)=\mathbf{0} .
$$

For

$$
\left(\mathbf{Q}-\kappa \mathbf{n} \mathbf{n}^{\mathrm{T}}\right) \geqslant 0, \quad \kappa>0,
$$

the existence and the uniqueness of the algebraic Riccati equation is guaranteed if the system is controllable and observable. 


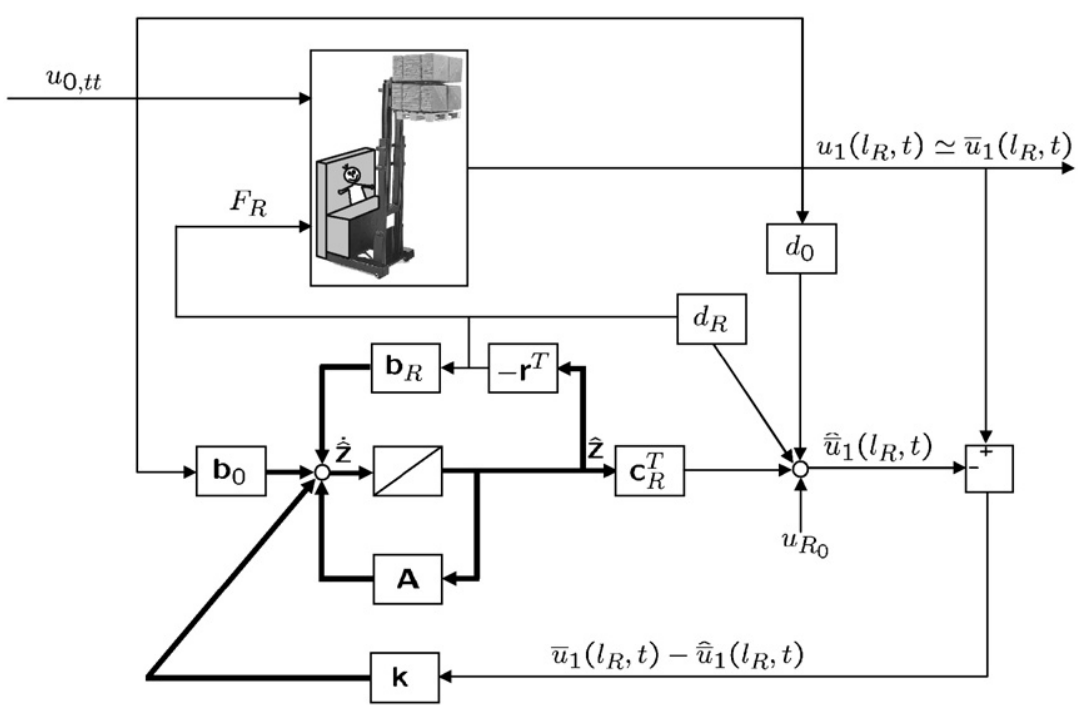

Fig. 7. Closed-loop system with state observer.

In this contribution, $\mathbf{Q}$ is chosen in such a way that $\mathbf{z}^{\mathrm{T}} \mathbf{Q z}$ represents the total energy (potential and kinetic energy) of the system, $\mathbf{n}$ is set to be zero

$$
\mathbf{Q}=\left(\begin{array}{cc}
\mathbf{\Omega}_{r} & \mathbf{0} \\
\mathbf{0} & \mathbf{I}
\end{array}\right), \quad \mathbf{n}=\mathbf{0}
$$

and the value of $\kappa$ is selected to achieve reasonably fast closed-loop poles without excessive values of the control effort [4].

\subsection{Full state observer}

The state feedback assumes that the states are known at all times. For the present problem, the state vector $\mathbf{z}$ cannot be measured directly, but the displacement $u_{1}\left(l_{R}, t\right)$ of the lower segment and the acceleration of the rigid vehicle unit $u_{0, t t}$ are measurable. The objective of a Luenberger Observer is to find from this information an approximate value $\hat{\mathbf{z}}$ of the state vector $\mathbf{z}$. The Luenberger Observer is written as (see Fig. 7)

$$
\dot{\hat{\mathbf{z}}}=\mathbf{A} \hat{\mathbf{z}}+\mathbf{b}_{R} F_{R}+\mathbf{b}_{0} u_{0, t t}+\mathbf{k}\left(\bar{u}_{1}\left(l_{R}, t\right)-\hat{\bar{u}}_{1}\left(l_{R}, t\right)\right),
$$

with

$$
\hat{\bar{u}}_{1}\left(l_{R}, t\right)=\mathbf{c}_{R}^{\mathrm{T}} \hat{\mathbf{z}}+d_{R} F_{R}+d_{0} u_{0, t t}+u_{R_{0}} .
$$

Combining the Eqs. (32)/(33) and (40)/(41), one obtains

$$
(\dot{\mathbf{z}}-\dot{\hat{\mathbf{z}}})=\left(\mathbf{A}-\mathbf{k c}_{R}^{\mathrm{T}}\right)(\mathbf{z}-\hat{\mathbf{z}}) .
$$

Eq. (42) shows that the error of the observer goes to zero if the eigenvalues of $\mathbf{A}-\mathbf{k c}_{R}^{\mathrm{T}}$ (observer poles) have negative real parts [4]. Due to the collocation of the actuator and of the sensor $\left(\mathbf{b}_{R}^{* *}=\mathbf{c}_{R}^{* *}\right)$, the observer poles correspond to the closed-loop poles (the eigenvalues of $\mathbf{A}-\mathbf{b}_{R} \mathbf{r}^{\mathrm{T}}$ ) for

$$
\mathbf{k}=\left(\begin{array}{ll}
\mathbf{0} & \mathbf{I} \\
\mathbf{I} & \mathbf{0}
\end{array}\right) \mathbf{r}
$$

To ensure that the observer is faster than the closed-loop, the observer poles are placed on the left of the closed-loop poles. Due to Eq. (43) this can be achieved by repeating the procedure illustrated in Section 4.3 using a new value $\kappa^{*}>\kappa$. Hence the regulator and the observer can both be designed from a single root locus. 


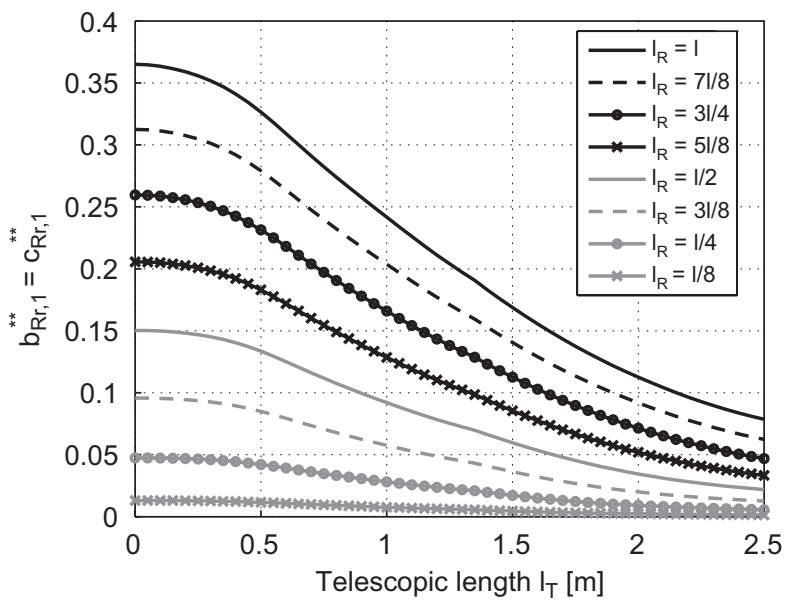

Fig. 8. First element of $\mathbf{b}_{R r}^{* *}$ and $\mathbf{c}_{R r}^{* *}$.

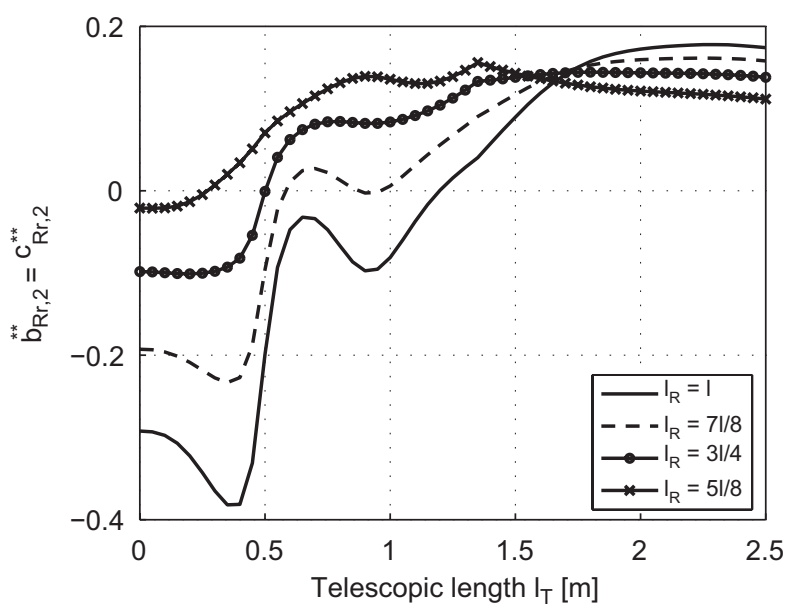

Fig. 9. Second element of $\mathbf{b}_{R r}^{* *}$ and $\mathbf{c}_{R r}^{* *}$ (not controllable).

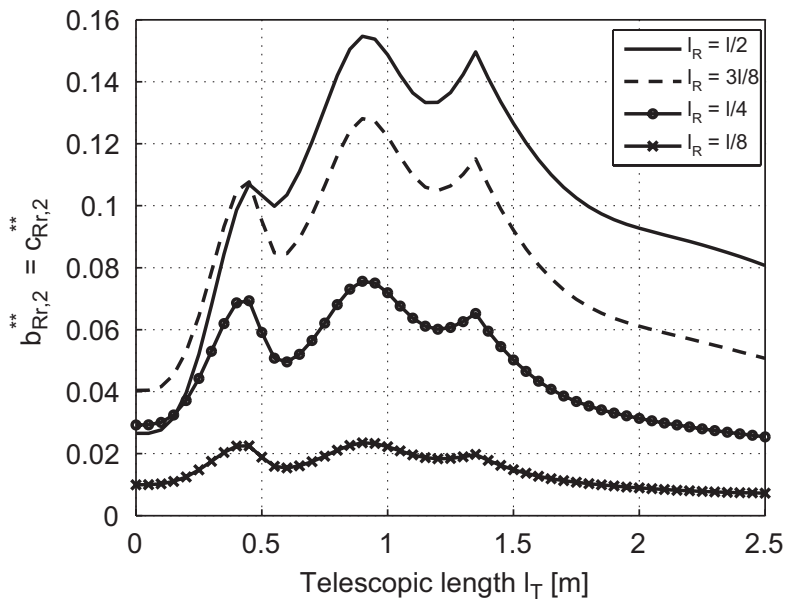

Fig. 10. Second element of $\mathbf{b}_{R r}^{* *}$ and $\mathbf{c}_{R r}^{* *}$ (controllable). 


\subsection{Telescopic operations with clearance}

For real telescopic operations, the parameters of the controller and of the observer are determined for different telescopic lengths $l_{T}=l_{L}+l_{A}$ (with $l_{A}=0$ for $0 \leqslant l_{L}<l$ ) and interpolation leads to an adaptive controller $\mathbf{r}\left(l_{T}\right)$ and an adaptive observer $\mathbf{A}\left(l_{T}\right), \mathbf{k}\left(l_{T}\right), \mathbf{b}_{R}\left(l_{T}\right), \mathbf{b}_{0}\left(l_{T}\right), \mathbf{c}_{R}\left(l_{T}\right), d_{R}\left(l_{T}\right), d_{0}\left(l_{T}\right), u_{R_{0}}\left(l_{T}\right)$. The position $z_{1}=l_{R}$ of the actuator-sensor pair has to be selected in such a way that the system is observable and controllable for any length $l_{T}$. This is fulfilled if the elements of $\mathbf{b}_{R r}^{* *}$ and $\mathbf{c}_{R r}^{* *}$, which coincide due to the collocation of the actuator-sensor pair, do not vanish. Figs. 8-10 show the developing of the elements of $\mathbf{b}_{R r}^{* *}$ and $\mathbf{c}_{R r}^{* *}$ versus the telescopic length $l_{T}$. In this example the system parameters are the same as in Section 4.1 with $N_{1}=N_{2}=4$ and $N_{r}=2$.

The developing of the first element of $\mathbf{b}_{R r}^{* *}$ and $\mathbf{c}_{R r}^{* *}$ (Fig. 8) shows that the first mode of the system is controllable and observable for each of the eight tested positions and that the controllability and observability increase with increasing values for $l_{R}$.

The second mode is not controllable or observable for each actuator-sensor position, which emanates from the zero-crossings of the plots of the second element of $\mathbf{b}_{R r}^{* *}$ and $\mathbf{c}_{R r}^{* *}$ shown in Fig. 9. A zero-crossing signifies that there is a nodal point of vibration of the second mode which coincides with the position of the actuator-sensor pair. The controllability and observability is only guaranteed for the four lowest positions and the best results are achieved choosing $z_{1}=l_{R}=l / 2$ as the position of the actuator-sensor pair (Fig. 10).

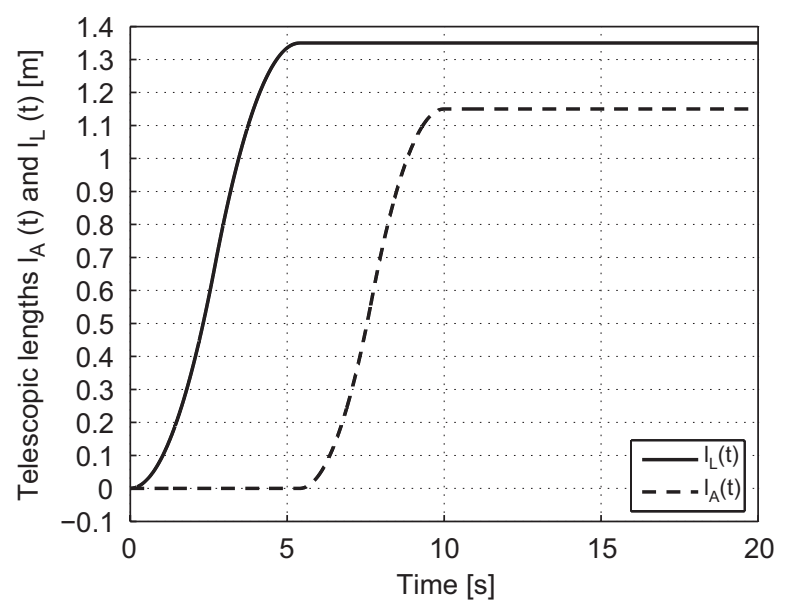

Fig. 11. Telescopic lengths $l_{A}(t)$ and $l_{L}(t)$.

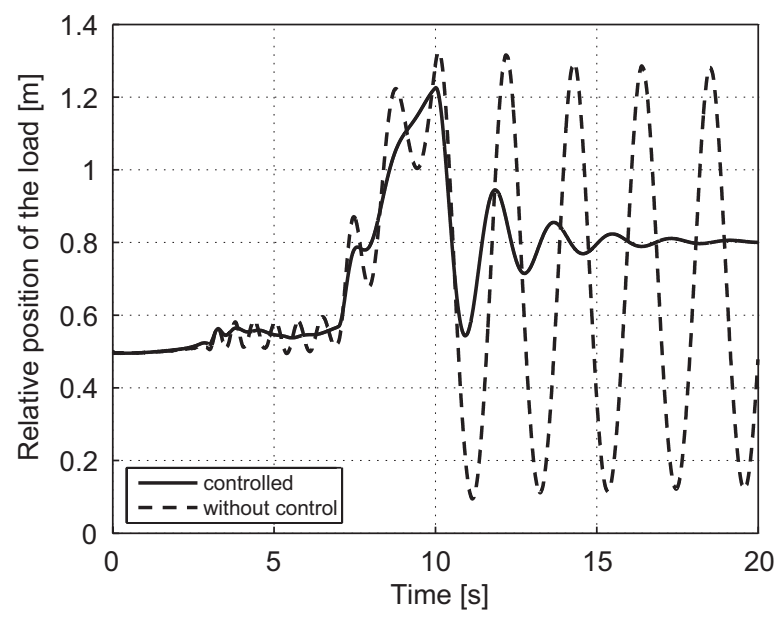

Fig. 12. Relative position of the load. 
First published in:

Due to the Luenberger Observer, straightforward measurements of the acceleration of the rigid vehicle unit $u_{0, t t}(t)$, of the telescopic length $l_{T}(t)$ and of the displacement $u_{1}\left(l_{R}, t\right)$ of the lower segment are sufficient to operate the controller. This allows to apply the adaptive controller and observer, developed for the reduced linear system model, to the original, significantly more complicated, geometrically nonlinear and time-varying system with clearance Eq. (22). Due to this approach, the influence of clearance and the robustness of the controlled system can be studied during dynamic telescopic motions.

\section{Simulation results and conclusions}

Results are presented here for a system with the parameters of Section 4.1 with $N_{1}=N_{2}=4, l_{S}=0.01 \mathrm{~m}$, $c=10^{8} \mathrm{~N} / \mathrm{m}$ and $d=10^{3} \mathrm{Ns} / \mathrm{m}$. The presented calculation example is similar to the example in Ref. [3] with the addition of the extending and retracting motion dynamics of the upper segment and of the load.

The system starts from an initial point without any initial velocity, is accelerated during $3 \mathrm{~s}\left(u_{0, t t}=5 \mathrm{~m} / \mathrm{s}^{2}\right)$ and moves with constant velocity during $4 \mathrm{~s}$ before it is decelerated during $3 \mathrm{~s}\left(u_{0, t t}=-5 \mathrm{~m} / \mathrm{s}^{2}\right)$. During the simulation the telescopic length $l_{T}=l_{A}+l_{L}$ increases as shown in Fig. 11. Fig. 12 shows the horizontal position $u_{2}\left(l_{L}(t), t\right)+a_{L} \cos \left(\alpha_{2}\left(l_{L}(t), t\right)\right)$ of the load relative to the vehicle unit during the motion and illustrates the vibration suppression by state control.

The present contribution shows that controlled vibration suppression of structural telescopic systems with clearance is possible, even during dynamic telescopic motions. For practical use, the dynamics of the actuator and of the sensor, which have been assumed to be perfect in this contribution, must be taken into account due to their possible destabilizing influence on the closed-loop. In real applications the influence of structural damping, which is neglected in this contribution, will improve the stability of the closed-loop.

\section{References}

[1] K. Fujita, Y. Shiona, K. Koike, T. Koseki, Multibody dynamics of the 2 section ladder model extending and retracting each other. Proceedings of ASME-DECT'03, Chicago, USA, September 2-6, 2003.

[2] P. Barthels, J. Wauer, Modeling and dynamic analysis of telescopic systems of structural members with clearance, Nonlinear Dynamics 42 (4) (2005) 371-382 ISSN: 0924-090X (Paper), 1573-269X (Online).

[3] P. Barthels, J. Wauer, Controlled vibration suppression of structural telescopic systems. IUTAM Symposium on Dynamics and Control of Nonlinear Systems with Uncertainty, Nanjing, China, Springer, IUTAM Bookseries, Vol. 2, 2006, pp. 287-296. ISBN: 978-1-40206331-2 (HB), 978-1-4020-6332-9 (e-book).

[4] A. Preumont, Vibration Control of Active Structures, second ed., Kluwer Academic Publishers, Dordrecht, 2002 ISBN 1-4020-0496-6. 